Original Research Paper

\title{
A Clean-Green Synthesis of Platinum Nanoparticles Utilizing a Pernicious Weed Lantana (Lantana Camara)
}

\author{
${ }^{1,2}$ Musthafa O. Mavukkandy, ${ }^{1}$ Sudip Chakraborty, ${ }^{2}$ Tasneem Abbasi and ${ }^{2}$ Shahid A. Abbasi \\ ${ }^{I}$ Centre for Pollution Control and Environmental Engineering, Pondicherry University, India \\ ${ }^{2} i$ Water, Masdar Institute of Science and Technology, UAE
}

Article history

Received: 13-01-2016

Revised: $15-01-2016$

Accepted: 25-01-2016

Corresponding Author:

Musthafa O. Mavukkandy

Centre for Pollution Control and

Environmental Engineering,

Pondicherry University, India and

iWater, Masdar Institute of

Science and Technology, UAE

Email: momavukkandy@masdar.ac.ae

\begin{abstract}
A rapid, reproducible single step method is proposed for synthesizing platinum nanoparticles using an invasive weed lantana (Lantana camara, $L$ ). The method involves mixing of platinum (VI) solution, lantana leaf extract and ascorbic acid in appropriate concentrations and keeping the mixture at $95^{\circ} \mathrm{C}$ for $8 \mathrm{~min}$. The collective action of ascorbic acid and leaf extract reduced the chloroplatinic acid to platinum nanoparticles and leaf extract then served the additional purpose of stabilizing the nanoparticles. The resulting nanoparticles have an average size of $35 \mathrm{~nm}$ and crystallized in face centred cubic symmetry. The course of the synthesis was trekked by UV-visible spectrophotometry and the characterization of the nanoparticles was done with scanning electron microscopy and confocal laser Raman spectroscopy. Particle size distribution was estimated by dynamic light scattering and the crystal structure was determined by XRD. The purity of the product was checked by EDAX.
\end{abstract}

Keywords: Biomimetic Synthesis, Green Synthesis, Platinum Nanoparticle, Weed Utilization

\section{Introduction}

Nanoparticles of noble metals, such as gold, silver and platinum are extensively used in the making of special shampoos, soaps, detergents, shoes, cosmetics and toothpastes. They also have widespread applications in drugs and drug delivery systems (Song et al., 2010). Platinum nanoparticles have been used in biomedical applications in blend with nanoparticles of other metals, in alloy, core-shell, or bimetallic nanocluster forms (Bhattacharya and Mukherjee, 2008). Yolk-shell nanocrystals of FePt@ $\mathrm{CoS}_{2}$ have been found to be highly effective in killing HeLa cells when compared to cis-platin (Gao et al., 2007). There is an excessive curiosity for synthesizing metal nanoparticles due to their fascinating properties which differ from their respective bulk counterparts (Mallikarjuna et al., 2011). Various physical and chemical techniques such as laser ablation, pyrolysis, lithography, chemical vapour deposition, sol-gel technique and electro-deposition have been employed for synthesizing nanoparticles, most of them being very expensive and use hazardous chemicals and produce toxic materials. These, along with vital biomedical applications necessitate the development of novel green syntheses approaches which do not involve the use of hazardous chemicals (Ankamwar et al., 2005; Roy and Barik, 2010). Biomimetic syntheses approaches utilizing microorganisms, live plants and plant extracts have been proposed as the possible eco-friendly, non-toxic alternatives to the conventional physical and chemical approaches (Kim et al., 2009; Mohanpuria et al., 2008).

Plant-based synthetic approaches have a multitude of benefits over other categories of biomimetic syntheses approaches, including the circumvention of tedious processes required to maintain the cell cultures; better control of the whole reaction; easy adaptability for scale up (Shankar et al., 2004) and tuning the size and shape in a desired manner (Iravani, 2011). Gold nanoparticles were synthesized within live alfalfa plants from solid media (Gardea-Torresdey et al., 2002). Extracellular nanoparticle synthesis employing plant parts would be more cost-effective when compared to the use of live plants since the downstream processing is easier in the former (Song et al., 2010). Recently several researchers pioneered the use of plant extracts to synthesize nanoparticles (Chandran et al., 2006; Rai et al., 2006; Shankar et al., 2003; 2004). While biomimetic 
approaches have been employed to synthesize nanoparticles of gold and silver, there is a dearth of literature concerning the biomimetic synthesis of platinum nanoparticles. It has been found that resting cells of Shewanella algae reduced aqueous $\mathrm{PtCl}_{6}$ into elemental platinum within $60 \mathrm{~min}$ under room temperature and neutral $\mathrm{pH}$ conditions when lactate was provided as an electron donor (Konishi et al., 2007). Synthesis of platinum nanoparticles using Diopyros kaki leaf extract was reported by (Song et al., 2010) in which he has shown the dependency of size on the reaction temperature and the concentrations of leaf broth and $\mathrm{PtCl}_{6}{ }^{2-}$. In another effort, wood nanomaterials in aqueous phase was used to synthesize platinum nanoparticles with controlled shapes and sizes (Lin et al., 2011).

In this study, we synthesized platinum nanoparticles using leaf extract of a pernicious weed Lantana camara, by which we succeeded in effecting double benefits viz., avoidance of toxic chemicals and the utilization of a noxious weed. We also investigated how nanoparticle synthesis was influenced by reaction conditions such as concentration of both leaf extract and the metal precursor solution $\mathrm{H}_{2} \mathrm{PtCl}_{6} \cdot 6 \mathrm{H}_{2} \mathrm{O}$, the effect of an external mild organic reducing agent, ascorbic acid, initiation temperature, duration of heating etc.

\section{Experimental}

Fresh Lantana camara (lantana) leaves were collected and the extract was prepared by boiling a mixture of $2 \mathrm{~g}$ dry weight equivalent of thoroughly washed fresh leaves and $100 \mathrm{~mL}$ of distilled water in a $300 \mathrm{~mL}$ beaker for $2 \mathrm{~min}$. The solution was decanted and stored at $4^{\circ} \mathrm{C}$ and was used within a week of having been prepared. Hexachloroplatinic acid was purchased from Sigma Aldrich. The concentrations of metal precursor ion and leaf extract were varied to explore the optimum combination. A mild organic reducing agent, ascorbic acid was used as an approach towards green synthesis, in order to reduce the duration of heating. All the chemicals used were of analytical grade. The resulting platinum nanoparticle solution was purified by repeated centrifugation at $12,000 \mathrm{rpm}$ for 15 min, with the precipitate produced by this process redispersed in deionized water.

For the synthesis of $\mathrm{Pt}$ nanoparticles, the mixture containing metal precursor $\mathrm{H}_{2} \mathrm{PtCl}_{6} \cdot 6 \mathrm{H}_{2} \mathrm{O}$, leaf extract and ascorbic acid was heated at $95^{\circ} \mathrm{C}$ for $8 \mathrm{~min}$. The solution turned into characteristic black colour, indicating the formation of Pt nanoparticles. The course of the synthesis was monitored by UV-visible spectrophotometry.

UV-Visible spectra were recorded as a function of the reaction time on an Analytical Technologies 'Spectro 2080 Plus' UV-Vis spectrophotometer, from 200 to $900 \mathrm{~nm}$.
The colloidal nanoparticle solution was centrifuged at $12000 \mathrm{rpm}$ for $15 \mathrm{~min}$, re-dispersed in deionized water, sonicated for 10 min using a Rivotek sonicator and then a thin film was made on the glass slide and used for the XRD analysis. The XRD spectra were recorded in PANalytical Xperto Pro X-ray Diffractometer and the X-ray diffracted intensities were recorded from 300 to 900 ( $2 \theta$ angles). The pattern was recorded using $\mathrm{Cu} K \alpha 1$ radiation with a wavelength $(\lambda)$ of $1.5406 \AA$ at a tube voltage of $40 \mathrm{kV}$ and a tube current of $30 \mathrm{~mA}$. The XRD data were analyzed using $X$ 'Pert High Score software for the identification of the crystalline phases. The crystal sizes of the synthesized Pt nanoparticle was calculated from the XRD spectra measurements by using the Scherrer's equation:

$$
D=\frac{K \lambda}{\beta \operatorname{Cos} \theta}
$$

where, $D$ corresponds to the crystal size, $K$ is the shapedependent Scherrer's constant, $\lambda$ is the wavelength of radiation, $\beta$ is the Full peak Width at Half-Maximum (FWHM) of the peak and $\theta$ is the Bragg diffraction angle (Sheny et al., 2011).

The capping or stabilizing compounds (agents), which help in stabilizing the particle, are of great interest in the case of bio-related applications, in describing the synthesis mechanism and in large scale production. Laser confocal Raman spectra analysis was done in a Renishaw Raman microscope to unveil the functional groups of compounds which were associated with nanoparticles. Preparation of nanoparticle samples/specimens was done using centrifugation for $15 \mathrm{~min}$ at $12000 \mathrm{rpm}$; which allow settling of nanoparticles at the bottom of the tube. Thin film of the samples were prepared after sonication for $10 \mathrm{~min}$, in a similar way for SEM analysis and were analyzed with a laser source of $514 \mathrm{~nm}$ in a range from 100-4000 $\mathrm{cm}^{-1}$. Acquisition time set was $30 \mathrm{~S}$.

Scanning electron micrographs were obtained using Quanta 200 FEG SEM in which samples were prepared by placing a drop of nanoparticle solution on a carbon tape pasted over a stub and allowing the sample to air dry. EDAX was done to analyze the purity of the particles by determining the elemental composition of particles which was determined using EDAX instrument (Energy-Dispersive X-ray Spectrometer (EDS; Thermo)) attached with the SEM instrument.

Average particle size and distribution were estimated by dynamic light spectroscopy. The centrifuged sample was re-suspended in distilled water and analyzed in Malvern zetasizer particle size analyzer. 


\section{Results and Discussion}

In the present study, various biomolecules present in the leaf extract of a pernicious terrestrial weed lantana were gainfully utilized both as a reducing and stabilizing agent in the synthesis of Pt nanoparticles. The reaction mixture containing the metal precursor solution and the leaf extract was heated at $95^{\circ} \mathrm{C}$ in a water bath for $3 \mathrm{~h}$ in order to synthesize Pt nanoparticles. A mild organic reducing agent, ascorbic acid was used in combination with leaf extract. It is done so in order to minimize the heating and thereby to make the process greener. The characteristic black colour of $\mathrm{Pt}$ nanoparticle was observed after heating at $95^{\circ} \mathrm{C}$ in water bath for $8 \mathrm{~min}$. Aggregation or precipitation of the formed metal nanoparticles was not observed for prolonged period $(>2$ months). Nanoparticle dispersion showed no significant change in their optical properties, indicating the excellent stabilizing action of the leaf extract.

The formation of $\mathrm{Pt}$ NPs was confirmed by a continuous absorption spectrum in the range of 200-900 $\mathrm{nm}$ in UV-visible spectrophotometer (Fig. 1). It can be seen that there is no distinct Surface Plasmon Resonance (SPR) occurs in this case and a continuous absorption is seen, which is in accordance with the earlier reports (Devi and Rao, 2000; Patel et al., 2005).

SEM micrographs of the synthesized Pt NPs (Fig. 2) shows a monodisperse spherical morphology.
Figure 3 shows the Energy Dispersive X-ray Analysis (EDAX) spectrum recorded in the spotprofile mode from one of the densely populated nanoparticles regions. Strong signals from the metal atoms in the nanoparticles are observed, while a strong signal of $\mathrm{Si}$ (from glass slide) and weaker signals from $\mathrm{C}, \mathrm{O}$ and $\mathrm{Na}$ atoms were also recorded.

The crystalline nature of $\mathrm{Pt}$ nanoparticles was confirmed from the X-ray diffraction analysis (Fig. 4). The typical XRD patterns of this nanoparticle thin film is shown in Fig. 3. The crystalline phases obtained with the formation of $\mathrm{Pt}$ nanoparticles could be indexed to $\left(\begin{array}{lll}1 & 1 & 1\end{array}\right),\left(\begin{array}{lll}2 & 0 & 0\end{array}\right),\left(\begin{array}{lll}2 & 2 & 0\end{array}\right)$ and $\left(\begin{array}{lll}3 & 1 & 1\end{array}\right)$ planes of facecentered cubic bulk metallic counterparts. A similar finding was reported by (Vinod et al., 2011) on the formation of $\mathrm{Pt}$ nanoparticles using a natural hydrocolloid gum kondagogu (Cochlospermum gossypium). The average crystal size was calculated by using Scherrer's equation and estimated as 9.93 nm. Confocal laser Raman spectroscopic measurements were carried out to identify the functional groups, which are specifically involved in the formation of metal nanoparticles (Fig. 5). The major functional groups present in the Raman spectrum are $-\mathrm{OH}, \mathrm{H}_{2} \mathrm{O}, \mathrm{CO}$. The average size of the synthesized Pt NPs were found to be $35 \mathrm{~nm}$ and the distribution is given in Fig. 6.

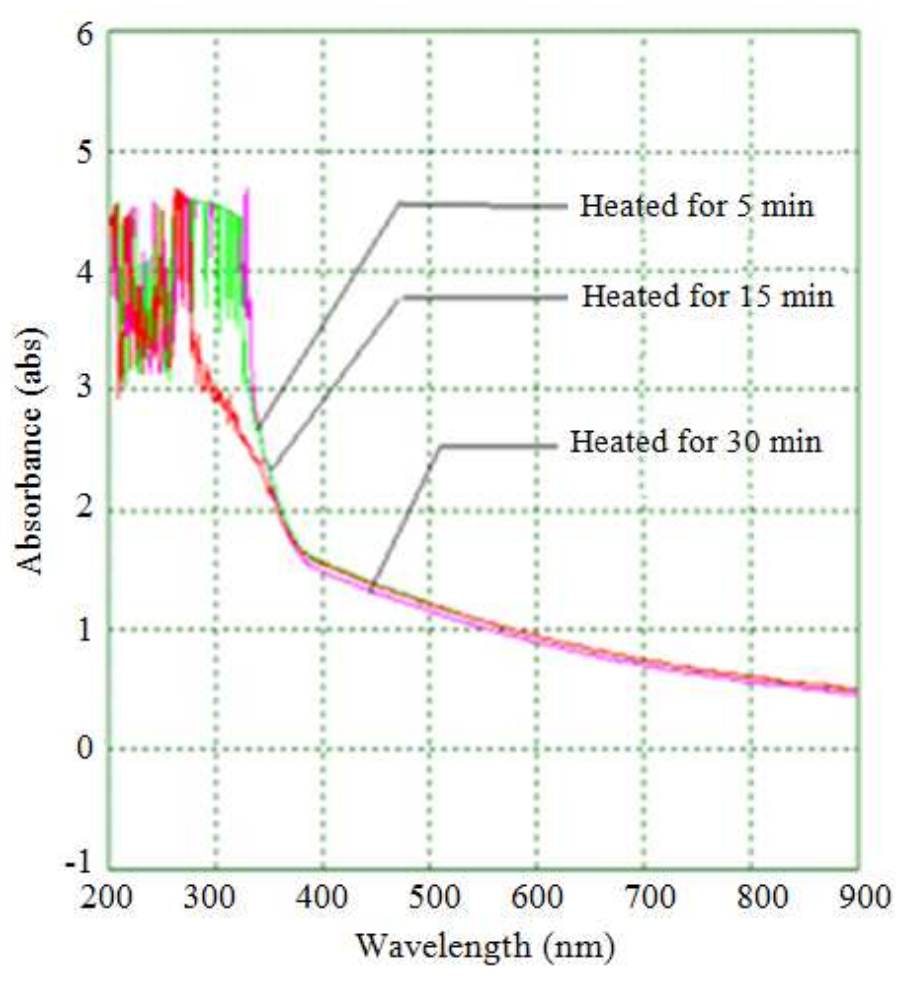

Fig. 1. UV-visible absorption spectra of Pt NPs synthesized 


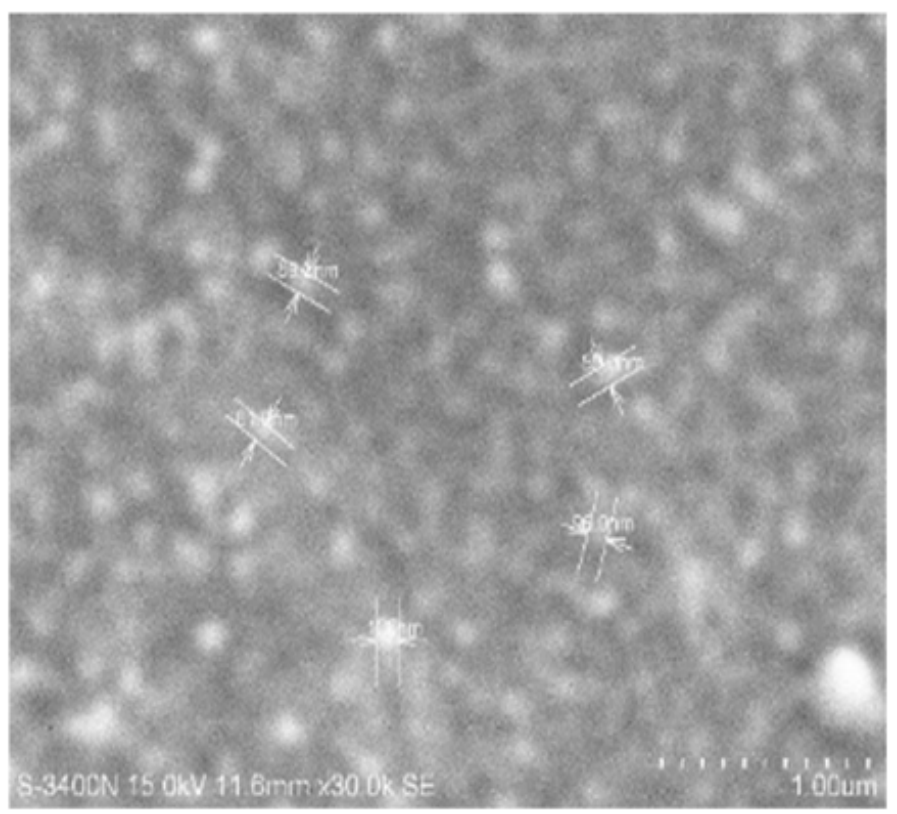

Fig. 2. SEM micrograph of synthesized Pt NPs

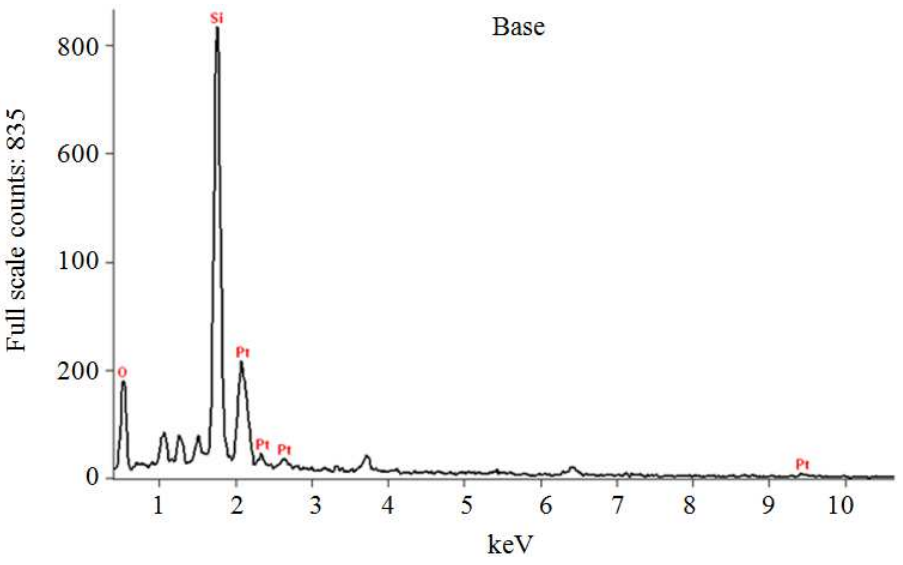

Fig. 3. EDAX profile of the synthesized Pt NPs

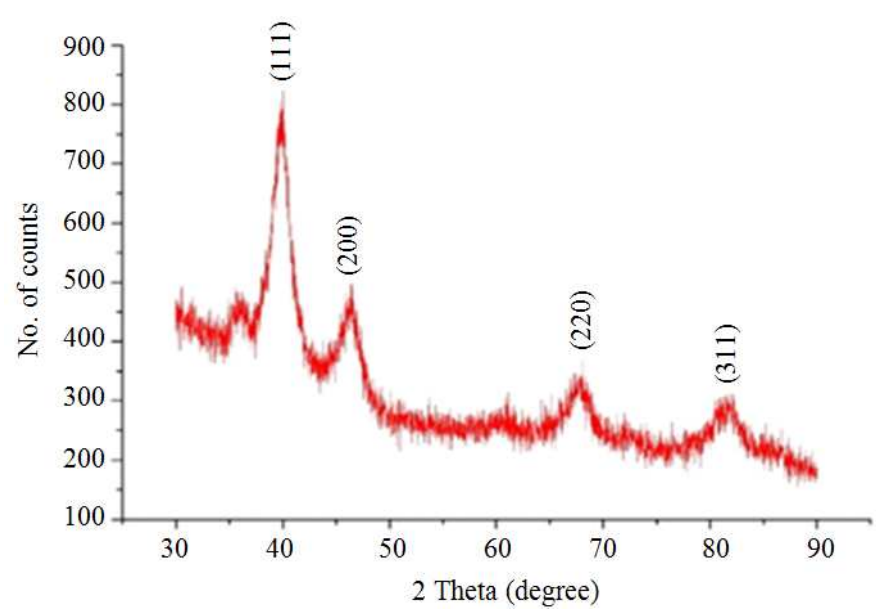

Fig. 4. XRD pattern of Pt nanoparticle showing its crystalline nature 


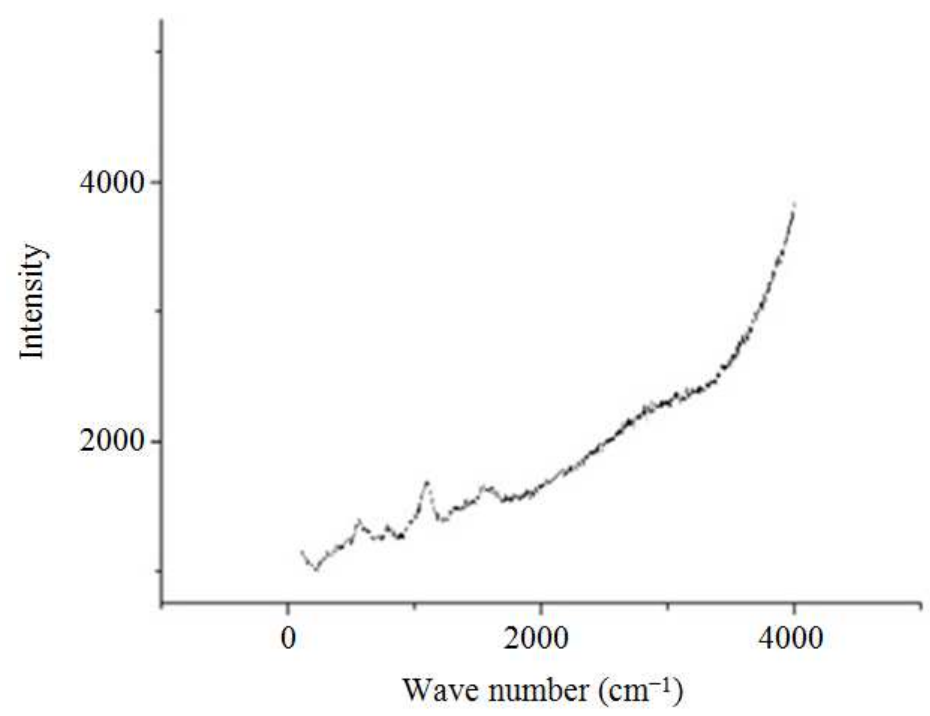

Fig. 5. Laser Raman spectrum indicating the crystal structure and functional groups
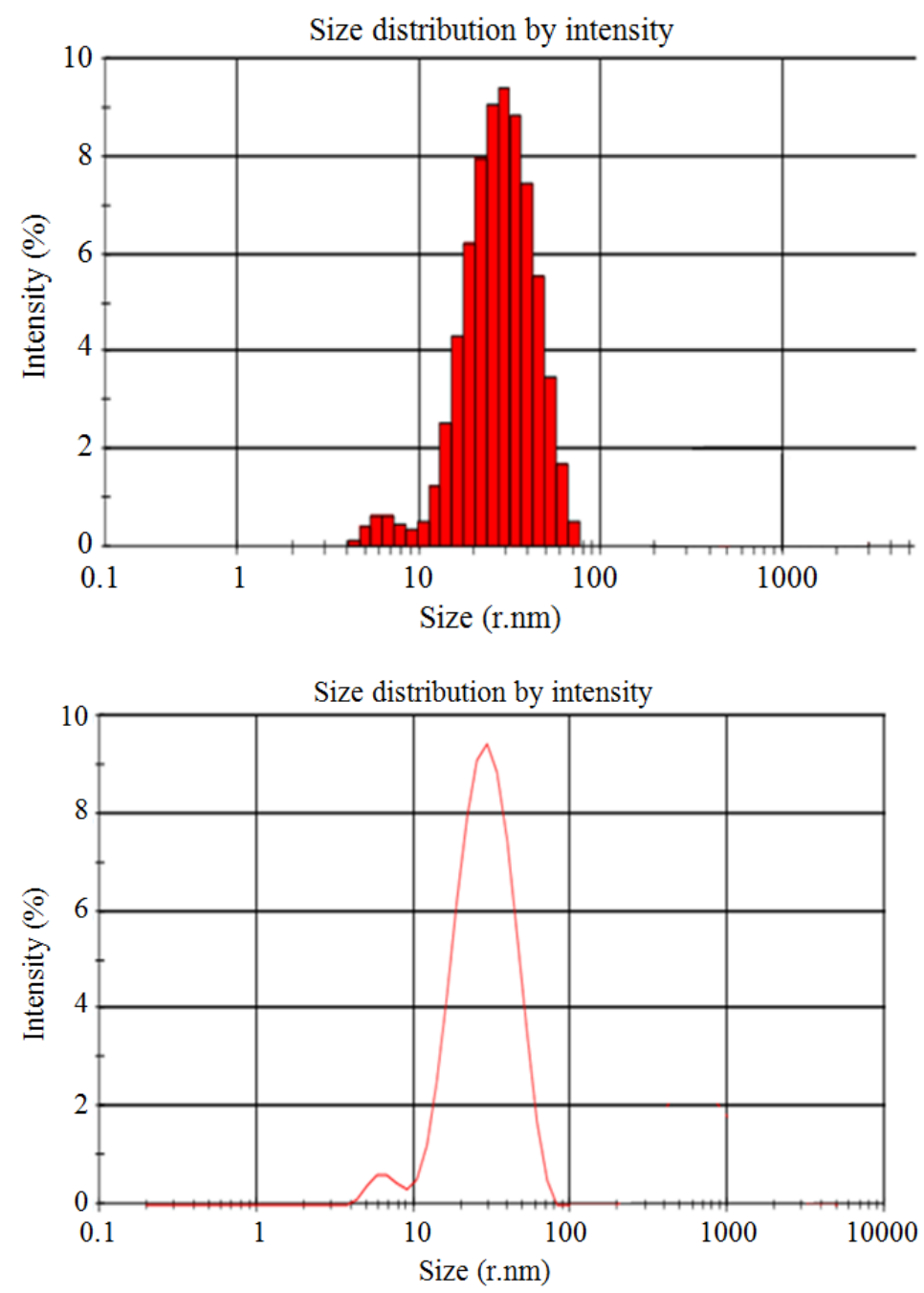

Fig. 6. Average particle size of the synthesized Pt NPs 


\section{Effects of Temperature and Reaction Mixture Composition}

The reduction of platinum ions to platinum nanoparticles when exposed to the leaf extract was followed by monitoring the changes in colour with UVVIS spectroscopy. It is found that an initial heating is essential for the NPs synthesis to occur. In order to see the possibility of avoiding the initial heating or to reduce the duration of heating, the prospect of using a mild organic reducing agent, ascorbic acid was explored. There was a significant reduction in the duration of heating: i.e., Pt NPs were formed by heating for $8 \mathrm{~min}$ at $100^{\circ} \mathrm{C}$ when compared to the $3 \mathrm{~h}$ in the absence of ascorbic acid. Prolonged heating at $100^{\circ} \mathrm{C}$ for more than $3 \mathrm{~h}$ has resulted in a lower OD, which may be attributed to the possible disintegration of the synthesized nanoparticles. Thus collective action of leaf extract and ascorbic acid (or any mild reducing agent) can enhance the rate of the reaction in a significant manner. There was no indication of nanoparticle synthesis when the reaction mixture was not heated, even though it contain a supplementary reducing agent, ascorbic acid. This implies that an initial heating is required in order to synthesize Pt nanoparticles. It is to be noted that when the concentration of external reducing agent, ascorbic acid, is higher than certain critical concentration, then the resulting OD is getting reduced, even though we can synthesize the NPs in a very short duration of heating (for e.g., $1 \mathrm{~min}$ ). Hence there should be a trade-off between the duration of heating and the concentration of the reducing agent in order to get an optimum yield of nanoparticles in a greener way.

\section{Effect of $p H$}

The characteristic black colour was not formed when the mixture $\mathrm{pH}$ was raised to neutral or alkaline. Thus it can be inferred that the acidic $\mathrm{pH}$ is favoured for the $\mathrm{Pt}$ NP synthesis.

\section{Effect of Various Proportions}

A leaf extract to the Pt metal ion ratio of 5:1 is found to be the optimum proportion. A higher concentration of extract inhibits the formation of Pt NPs, which is in accordance with the earlier observation (Bonet et al., 1999) where they have reported that in the case of Pt, when the concentration of stabilizing agent, PVP, increases, formation of NPs got inhibited.

\section{Conclusion}

For the first time, platinum nanoparticles were synthesized from its precursor chloroplatinic acid (H2PtCl6) utilizing a pernicious terrestrial invasive weed lantana (Lantana camara) as the reducing and stabilizing agent. The addition of a mild organic reducing agent, ascorbic acid helped to reduce the duration of heating from $3 \mathrm{~h}$ to $8 \mathrm{~min}$, thus making the process greener. The aqueous leaf extract of lantana allowed the rapid formation of Pt nanoparticles with an average size of 35 $\mathrm{nm}$ with a spherical shape.

\section{Acknowledgement}

We thank the Central Instrumentation Facility (CIF) and the Dept. of Physics, Pondicherry University for their help in doing characterization such as SEM, EDAX, DLS, CLRS and XRD, Mr. Sami U. Ganaie for his comments.

\section{Author's Contributions}

All the authors except the second author have equal contribution in preparation of the manuscript. The second author did the data analysis and organization of the manuscript including language corrections and review.

\section{Ethics}

Authors should address any ethical issues that may arise after the publication of this manuscript.

\section{References}

Ankamwar, B., M. Chaudhary and M. Sastry, 2005. Gold nanotriangles biologically synthesized using tamarind leaf extract and potential application in vapor sensing. Synthesis Reactivity Inorganic Metal-Organic Nano-Metal Chem., 35: 19-26.

DOI: $10.1081 /$ SIM-200047527

Bhattacharya, R. and P. Mukherjee, 2008. Biological properties of "naked" metal nanoparticles. Adv. Drug Delivery Rev., 60: 1289-1306.

DOI: 10.1016/j.addr.2008.03.013

Bonet, F., V. Delmas, S. Grugeon, R. Herrera Urbina and P.Y. Silvert et al., 1999. Synthesis of monodisperse $\mathrm{Au}, \mathrm{Pt}, \mathrm{Pd}, \mathrm{Ru}$ and $\mathrm{Ir}$ nanoparticles in ethylene glycol. Nanostructured Mater., 11: 1277-1284. DOI: 10.1016/S0965-9773(99)00419-5

Chandran, S.P., M. Chaudhary, R. Pasricha, A. Ahmad and M. Sastry, 2006. Synthesis of gold nanotriangles and silver nanoparticles using Aloevera plant extract. Biotechnol. Progress, 22: 577-583. DOI: $10.1021 / \mathrm{bp} 0501423$

Devi, G.S. and V.J. Rao, 2000. Room temperature synthesis of colloidal platinum nanoparticles. Bull. Mater. Sci., 23: 467-470. DOI: 10.1007/BF02903885

Gao, L., J. Zhuang, L. Nie, J. Zhang and Y. Zhang et al., 2007. Intrinsic peroxidase-like activity of ferromagnetic nanoparticles. Nat. Nanotechnol., 2: 577-583. DOI: $10.1038 /$ nnano. 2007.260 
Gardea-Torresdey, J.L., J.G. Parsons, E. Gomez, J. Peralta-Videa and H.E. Troiani et al., 2002. Formation and growth of $\mathrm{Au}$ nanoparticles inside live alfalfa plants. Nano Lett., 2: 397-401. DOI: $10.1021 / \mathrm{nl} 015673+$

Iravani, S., 2011. Green synthesis of metal nanoparticles using plants. Green Chem., 13: 2638-2650. DOI: $10.1039 / \mathrm{C} 1 \mathrm{GC} 15386 \mathrm{~B}$

Kim, C.K., P. Ghosh and V.M. Rotello, 2009. Multimodal drug delivery using gold nanoparticles. Nanoscale, 1: 61-67. DOI: 10.1039/B9NR00112C

Konishi, Y., K. Ohno, N. Saitoh, T. Nomura and S. Nagamine et al., 2007. Bioreductive deposition of platinum nanoparticles on the bacterium Shewanella algae. J. Biotechnol., 128: 648-653. DOI: 10.1016/j.jbiotec.2006.11.014

Lin, X., M. Wu, D. Wu, S. Kuga and T. Endo et al., 2011. Platinum nanoparticles using wood nanomaterials: Eco-friendly synthesis, shape control and catalytic activity for p-nitrophenol reduction. Green Chem., 13: 283-287. DOI: 10.1039/C0GC00513D

Mallikarjuna, K., G. Narasimha, G.R. Dillip, B. Praveen and B. Shreedhar et al., 2011. Green synthesis of silver nanoparticles using Ocimum leaf extract and their characterization. Digest J. Nanomater. Biostructures, 6: 181-186.

Mohanpuria, P., N.K. Rana and S.K. Yadav, 2008. Biosynthesis of nanoparticles: Technological concepts and future applications. J. Nanoparticle Res., 10: 507-517. DOI: $10.1007 / \mathrm{s} 11051-007-9275-\mathrm{X}$

Patel, K., S. Kapoor, D. Dave and T. Mukherjee, 2005. Synthesis of $\mathrm{Pt}, \mathrm{Pd}, \mathrm{Pt} / \mathrm{Ag}$ and $\mathrm{Pd} / \mathrm{Ag}$ nanoparticles by microwave-polyol method. J. Chem. Sci., 117: 311-316. DOI: 10.1007/BF02708443
Rai, A., A. Singh, A. Ahmad and M. Sastry, 2006. Role of halide ions and temperature on the morphology of biologically synthesized gold nanotriangles. Langmuir, 22: 736-741. DOI: 10.1021/la052055q

Roy, N. and A. Barik, 2010. Green synthesis of silver nanoparticles from the unexploited weed resources. Int. J. Nanotechnol. Applic., 4: 95-101.

Shankar, S.S., A. Ahmad and M. Sastry, 2003. Geranium leaf assisted biosynthesis of silver nanoparticles. Biotechnol. Progress, 19: 1627-1631. DOI: $10.1021 / \mathrm{bp} 034070 \mathrm{w}$

Shankar, S.S., A. Rai, A. Ahmad and M. Sastry, 2004. Rapid synthesis of $\mathrm{Au}, \mathrm{Ag}$ and bimetallic Au coreAg shell nanoparticles using Neem (Azadirachta indica) leaf broth. J. Colloid Interface Sci., 275: 496-502. DOI: 10.1016/j.jcis.2004.03.003

Sheny, D.S., J. Mathew and D. Philip, 2011. Phytosynthesis of $\mathrm{Au}, \mathrm{Ag}$ and $\mathrm{Au}-\mathrm{Ag}$ bimetallic nanoparticles using aqueous extract and dried leaf of Anacardium occidentale. Spectrochimica Acta, 79: 254-262. DOI: 10.1016/j.saa.2011.02.051

Song, J.Y., E.Y. Kwon and B.S. Kim, 2010. Biological synthesis of platinum nanoparticles using Diopyros kaki leaf extract. Bioprocess Biosyst. Eng., 33: 159-164. DOI: 10.1007/s00449-009-0373-2

Vinod, V.T.P., P. Saravanan, B. Sreedhar, D.K. Devi and R.B. Sashidhar, 2011. A facile synthesis and characterization of $\mathrm{Ag}, \mathrm{Au}$ and $\mathrm{Pt}$ nanoparticles using a natural hydrocolloid gum kondagogu (Cochlospermum gossypium). Colloids Surfaces B, 83: 291-298. DOI: 10.1016/j.colsurfb.2010.11.035 